\title{
Midterm Follow up Analysis of the Functional And Radiological Outcome of Un-Cemented Hydroxyapatite Coated Stem in Total Hip Arthroplasty
}

\author{
Dr. J. Dhinesh ${ }^{1}$,Dr. M. Mohan Kumar $^{2}$,Dr. Pravin K Vanchi ${ }^{3}$ \\ ${ }_{1,2,3}$ (Department Of Orthopaedics Sriramachandra University, Chennai India
}

\begin{abstract}
Our study is a retrospective one analyzing the functional and radiological outcome of un-cemented Hydroxyapatite coated stem in Total Hip Arthroplasty. The total number of hips in our study was 40 all of them done by a single surgeon. With a mean follow up of 8.075 years. The average age of all the patients was 56.35 years. The prosthesis used was a standard collarless corail stem (Depuy) with Duraloc shell. The clinical evaluation was done using Harris hip score. Standard $x$-rays were taken to analyze the radiological outcome. $85 \%$ of our patients had an excellent result on the Harris hip score and $12.5 \%$ of our patients had a good result. The mean modified Harris hip score was $96.2 \%$ during the midterm follow up. 5\% of our patients had limb lengthening between 1-1.5cm with good modified Harris hip score. Radiological evaluation was done $5 \%$ of our patients had a Varus stem position, $85 \%$ of them had a central stem position. None of the patients had any femoral loosening. Vertical subsidence of negligible $3-5 \mathrm{~mm}$ was seen in $7.5 \%$ of our patients with no change in clinical outcome. None of our patients had heterotrophic ossification. 5\% of our patients had periosteal bone remodeling reactions. Our study concludes that Un-cemented primary Hydroxyapatite coated Total Hip Arthroplasty is a reliable procedure with excellent implant fixation and a predictable clinical outcome. Modified Harris hip score is a useful scoring system in assessing Total Hip Arthroplasty.
\end{abstract}

Keywords: Hip surgeries, Hydroxyapatite coated stem, Total hip Arthroplasty, Un-cemented stem

\section{Introduction}

The hip joint forms the connection between the lower limb and pelvic girdle. The hip joint is designed for stability as well as for a wide range of movements. This multi-axial ball and socket joint allows the entire lower extremity to move in three planes of motion, while providing an important shock absorption function to the torso and upper body.

Pain in the hip joint is one of the most important causes in disabling the human locomotion. Pain in the hip joint may be due to various causes ranging from fracture neck of femur in the elderly to rheumatoid arthritis in the younger age. There are many ways and methods by which this crippling pain in the hip can be treated. This includes analgesics, using a walking stick or bilateral axillary crutches, arthrodesis, and total hip Arthroplasty.

Arthroplasty is an operation to restore motion and stability to a joint and function to the muscle, ligaments and other soft tissue structures that control the joint. Implanting an artificial head and socket to replace the degenerated head exerted such a profound social impact and enjoyed such a dramatic early success.

Various immediate and long term complications may compromise this procedure, but it still remains the greatest boon available to Orthopaedic patients, and has proved to be the greatest advancement in the field of Orthopaedic surgery in the twentieth and twenty first century. Every surgeon who thinks about doing a primary total hip replacement should always plan ahead and indeed be capable of revising his surgery. In other words doing a total hip replacement should not be taken lightly.

The inclination of the modem day Orthopaedic surgeon is to do a cement less fixation, which is thought to be less morbid than the conventional cemented total hip Arthroplasty.

In our center we have the facility to do both cemented and un-cemented type of total hip Arthroplasty. We have decided to study the midterm follow up of un-cemented Total hip Arthroplasty done in our center during the last 10 years in patients for various indications.This is a single center, where in multiple surgeons and many techniques have been used amounting to a multicenter case collection in its nature. We thought we would review our un-cemented total hip replacements done during the last 10 years and analyze the radiological changes and its correlation with the clinical presentation.

\section{Aim}

Our study is a retrospective one analyzing the functional and radiological outcome of un-cemented Hydroxyapatite coated stem in Total Hip Arthroplasty 


\section{Materials And Methods}

This is a retrospective study done by a single surgeon in a single center. The total number of patients was 39 and the total number of hips operated was 40 ( 1 patient had a bilateral hip replaced). The follow up period was for a minimum of 7 years to a maximum of 9 years. The mean follow up period was 8.075 years. The mean age group of patients was 56.35 years with the youngest as low as 26 years and the oldest 74 years. 24 patients were male and 15 were female. 26 patients had a neck of femur fracture, 9 patients had avascular necrosis of the femoral head, 2 patients had rheumatoid arthritis (Fig:1). All patients were fixed with a standard collarless coral stem (Depuy) with Duraloc Cup. Post-operatively in our study we have used the modified harris hip score to assess the functional outcome. The surgical technique and post-operative protocol followed for all the patients were the same and it was a single surgeon single center study. The radiological evaluation included a number of factors as described below in the results to assess the fixation with collarless stems.

Fig: 1

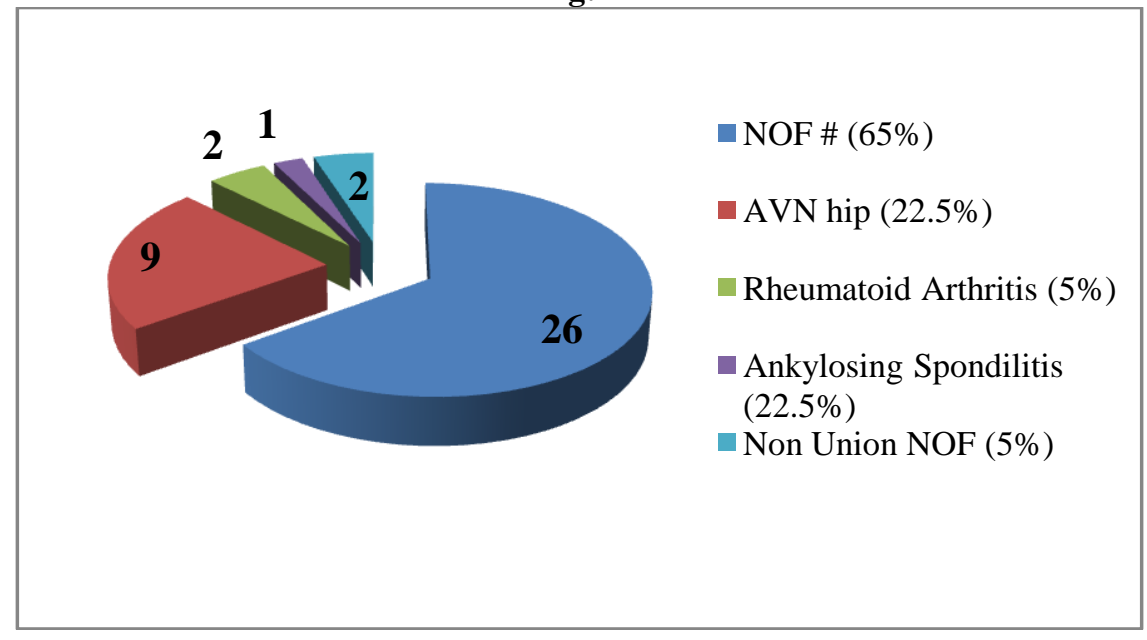

\subsection{Clinical Evaluation:}

\section{Observations and Results}

4.1.1 Modified harris hip score: Clinical evaluation was done 7-9 years of post op period using Modified Harris hip score which takes into account pain, function, deformity and range of movements.85\% of our patients had excellent results, while $12.5 \%$ patients had a good modified Harris hip score. 1 patient $2.5 \%$ had a poor result (Fig:2)

Fig:2 Modified Harris hip score

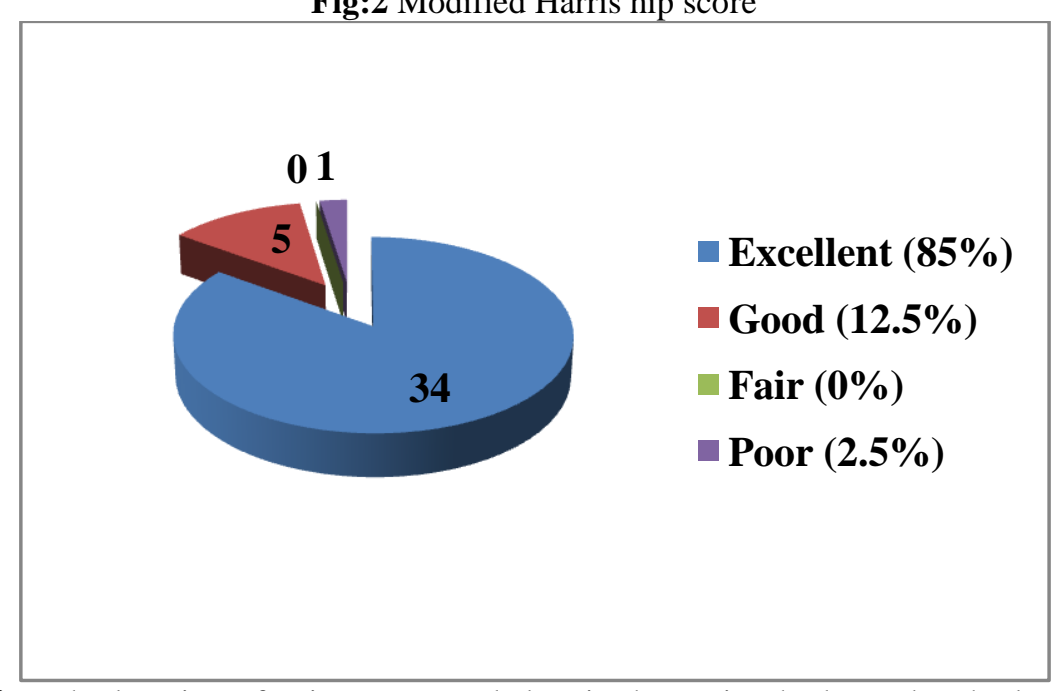

4.1.2 Pain: The location of pain was recorded as in the groin, the buttocks, the lateral or trochanteric area, the anterior aspect of the thigh or diffuse. At the latest follow up visit, 33 hips had a score of 44 points; 6 hips had 40 points; 0 hip had 30 points; 1 hip had 20 points; 0 hip had 10 points; 0 hip had 0 points. (Fig:3) 
Fig:3 Pain Assessment

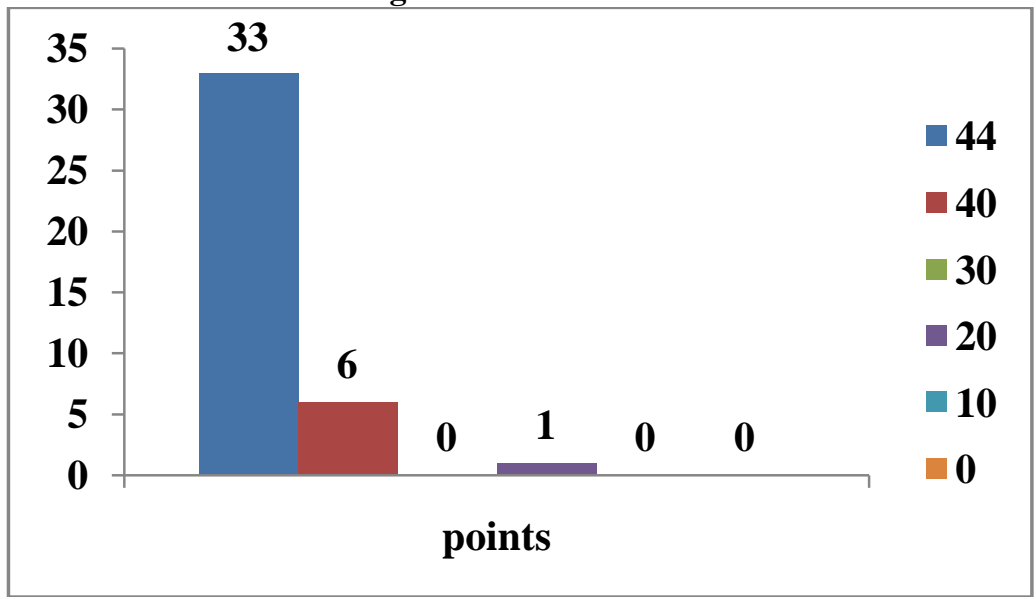

4.1.3. Limp: At the latest follow up visit 39 hips had a score of 11 points; 0 hip had 8 points; 1 hip had 5 points; 0 hip had 0 points.

4.1.4. Support (Walking aids): At the latest follow up visit 33 hips had 11 points; 6 hips had 7 points; 6 hips had 5 points; 0 hip had 3 points; 0 hip had 2 points; 0 hip had 0 points. (Fig:4)

4.1.5: Walking Distance: At the latest follow up 33 hips had 11 points 5 hips had 8 points; 2 hips had 5 points; 0 hip had 2 points; 0 hip had 0 points.

Fig:4

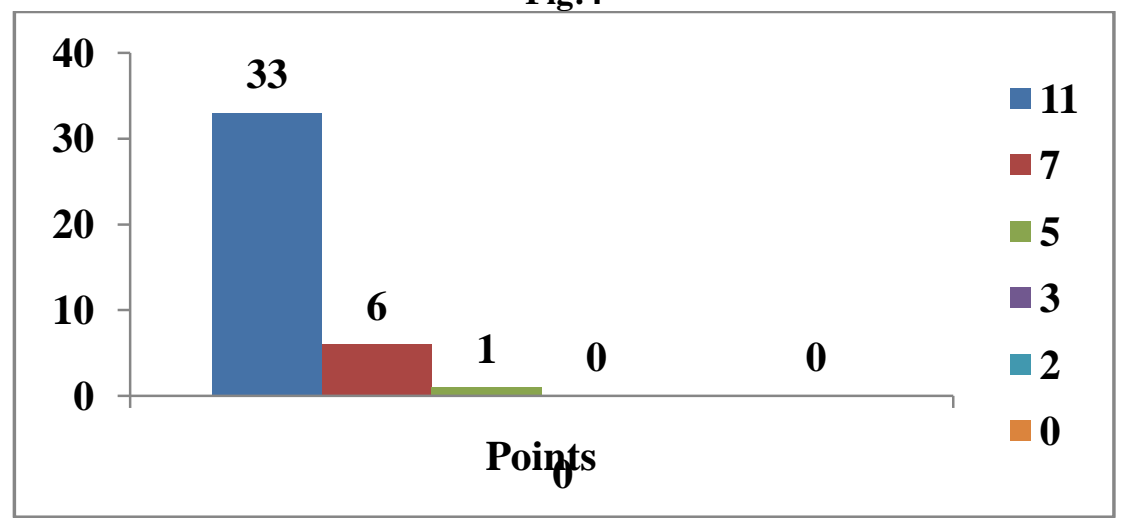

4.1.6. Stair Climbing: At the latest follow up 36 hips had 4 points; 3 hips had 2 points; 1 hip had 1 point; 0 hip had 0 points.

4.1.7. Range of Motion: At the latest follow up 30 hips had 5 points; 5 hips had 4 points; 4 hips had 3 points; 0 hip had 2 points; 1 hip had 1 point, 0 hip had 0 point.

4.1.8. Sitting: At the latest follow up 38 hips had 5 points; 2 hips had 3 points; 0 hip had 0 points. (Fig:5)

Fig:5

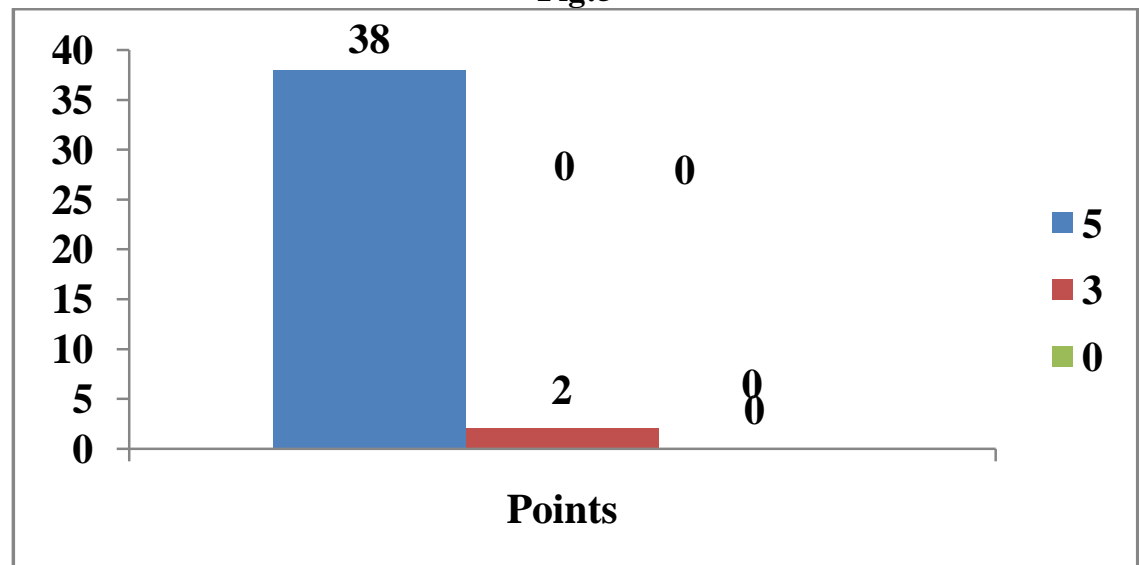

4.1.9. Activities: At the latest follow up 37 hips had 4 points; 3 hips had 2 points; 0 hip had 0 points. 4.1.10. Public Transportation: 39 hips able to use public transportation at the latest follow up. 
4.1.11. Deformity: Before the operation 2 hips had fixed flexion deformity, while others hips had no deformities. Post operatively there were no deformities in any of the patients.

\subsubsection{Modified Harris Hip Score in Follow Up (Fig: 6)}

- Mean 7 years: $96.3 \%$

- Mean 8 years: $94.8 \%$

- $\quad$ Mean 9 years: $97.7 \%$

Mean overall years $96.2 \%$

Fig:6 Modified Harris Hip score

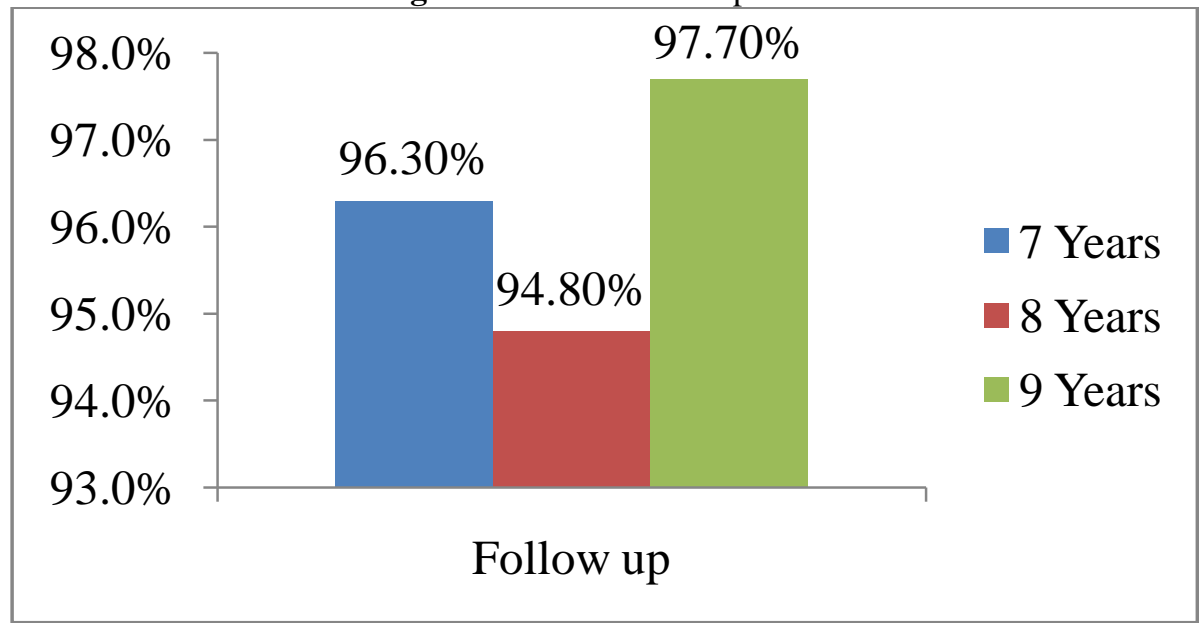

4.1.13. Limb Length Discrepancy:(Fig:7)

In this study 2 patients had limb lengthening

- Both of them were corrected using heel rise

- Modified Harris hip score were good in both the cases.

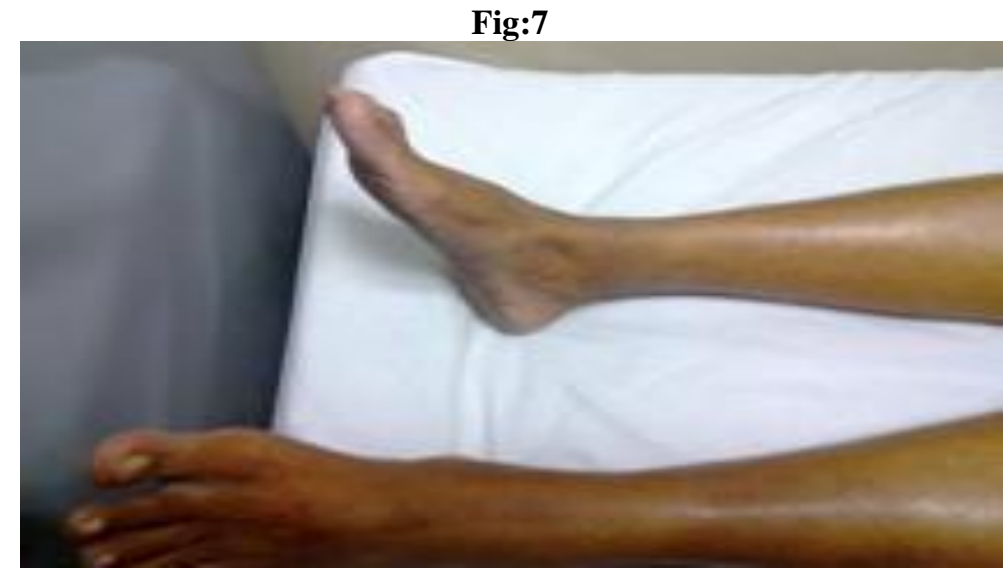

\subsection{Radiological Evaluation}

Observations and measurements were made on the antero posterior radiograph of the pelvis and on the anteroposterior and lateral radiograph of the hip. Radiographic evaluation included

1. Loosening of the Femoral components.

2. Femoral Stem Position

3. Vertical Subsidence

4. Endosteal Bone Apposition

5. Heterotopic Ossification

6. Bone Reactive Lines

7. Periosteal Bone Reaction

8. Pedestal Formation

9. Calcar Resorption 
10. Osteoporosis

11. Osteolysis

12. Polyethylene Wear

\subsubsection{Loosening of the Femoral Components: We have no cases of femoral loosening.}

4.2.2. Femoral Stem Position: The position of the femoral component in the frontal plane was measured on the anteroposterior radiographs, as previously described. In this study the results were as follows; $85 \%$ of our patients who underwent un-cemented total hip replacement had stem position in center. $10 \%$ of our patients had valgus stem. $5 \%$ patients had varus stem position at the latest position.(Fig: 8,9,10)

- Centre - 34(85\%)

- Valgus - $4(10 \%)$

- Varus - $2(5 \%)$

Fig: 8

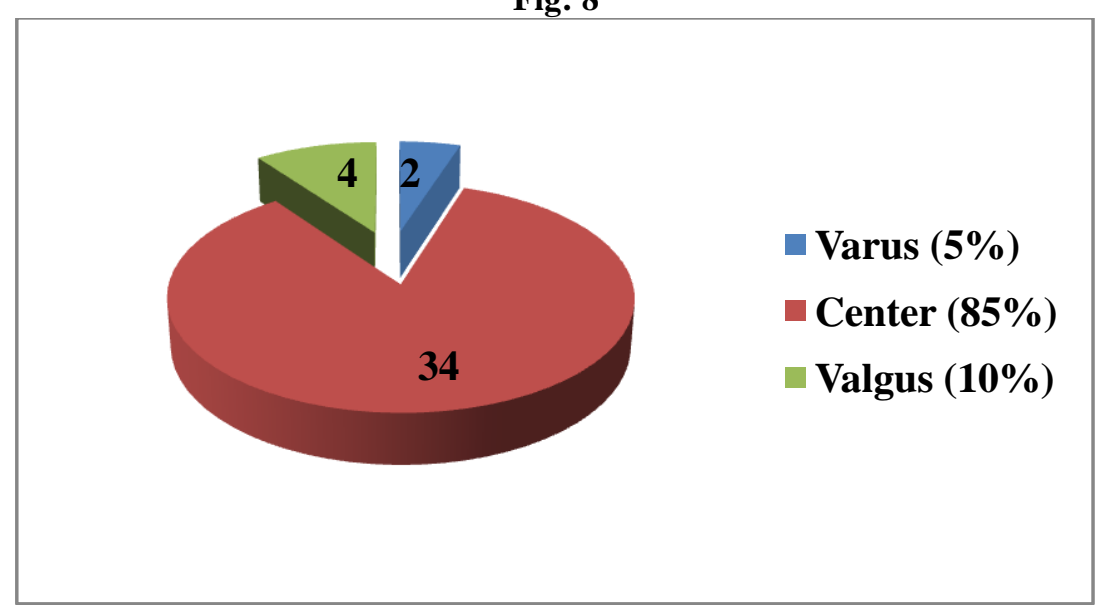

Fig:9: Et 26/M left hip AVN stage-3,Modified HHS-88

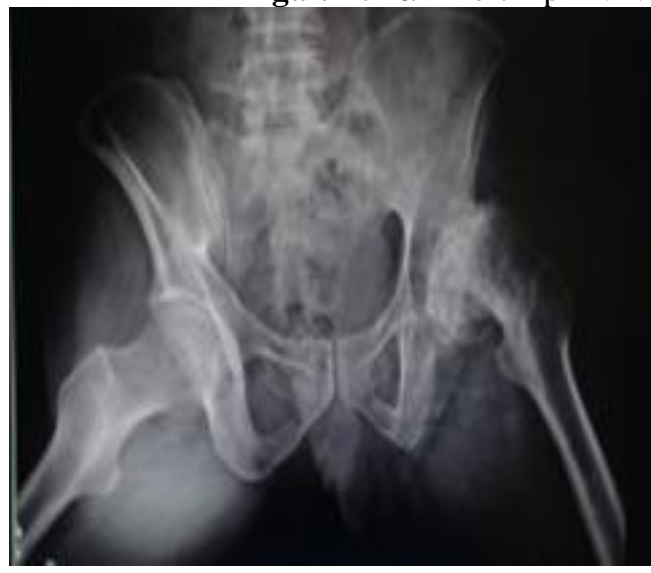

Pre-operative

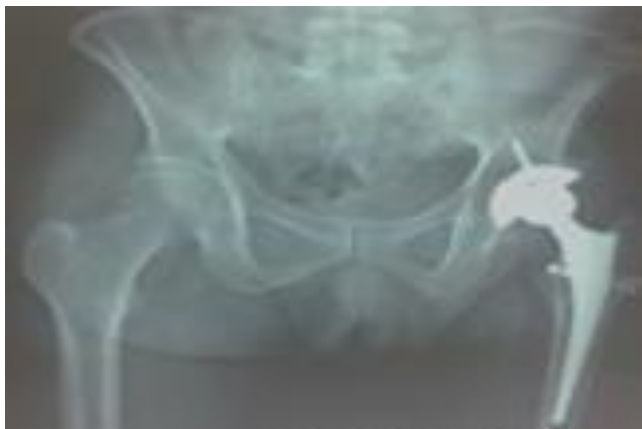

4 years post op

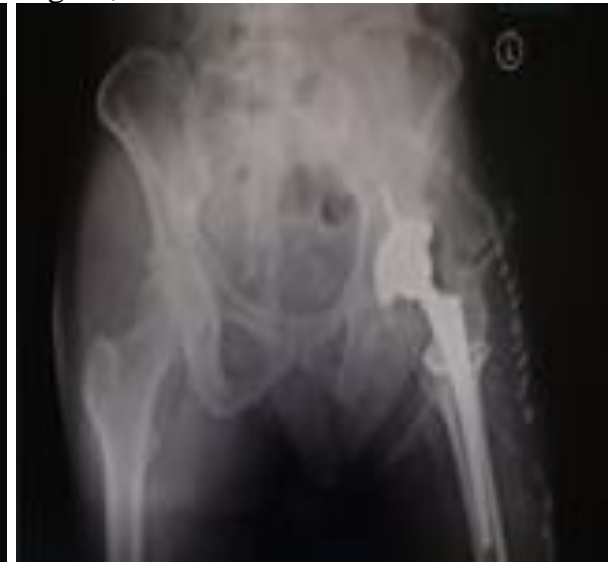

Post op

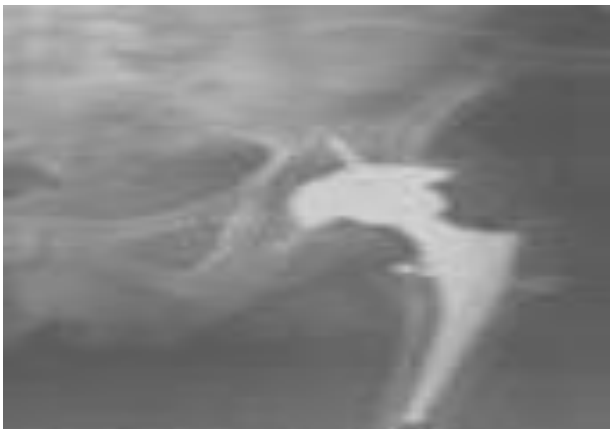

7 years post op showing valgus stem 
Fig: 10: Cha 64/M Left NOF\#,modified HHS-97

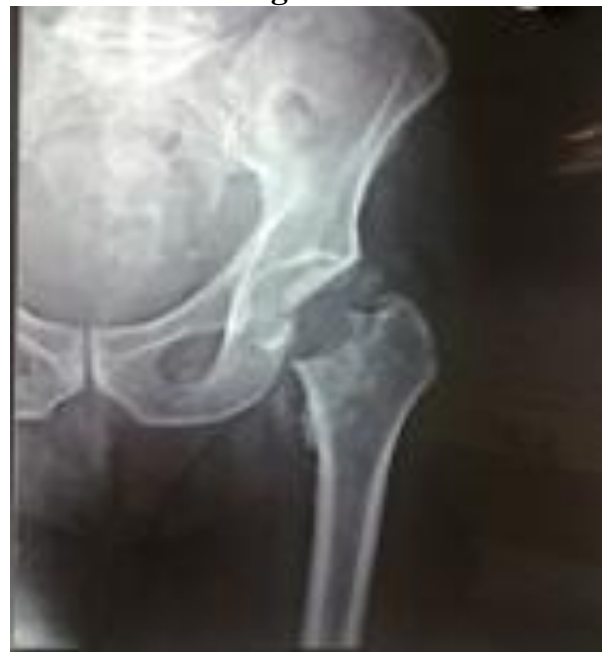

Pre-operative

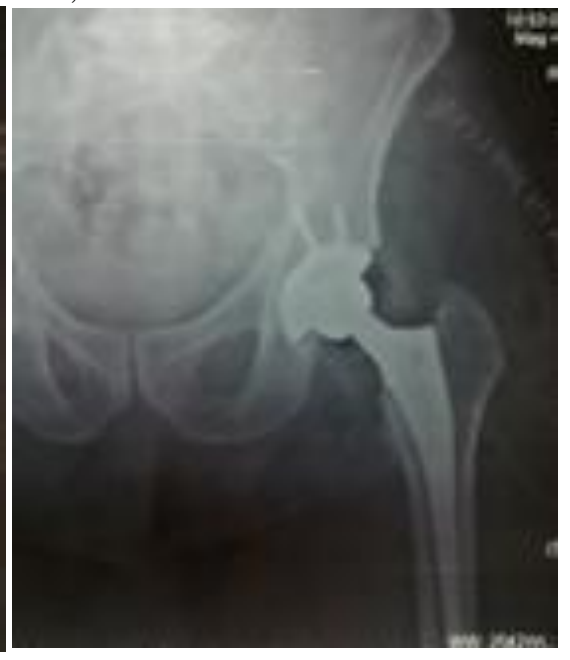

Immediate Post op

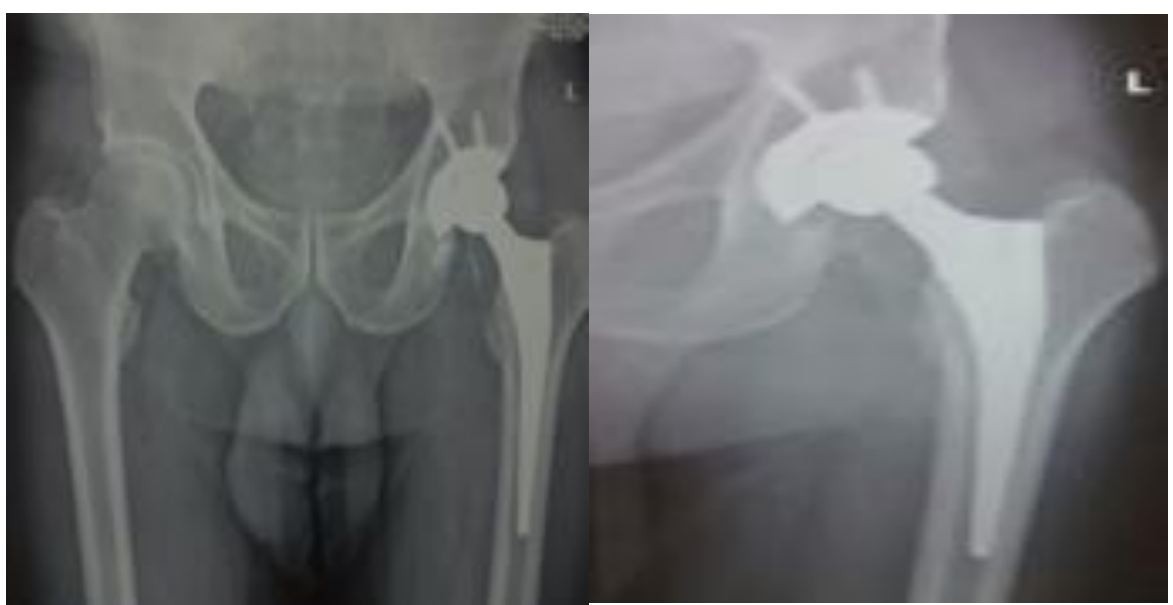

4 years post op

8 years post op showing varus stem

\subsubsection{Vertical Subsidence}

Vertical subsidence of the femoral component was measured on the anteroposterior radiographs as previously described. In this latest follow up 3 patients had vertical subsidence with minimum of $3 \mathrm{~mm}$ to a maximum of $5 \mathrm{~mm}$. there is no change in the clinical outcome of these 3 patients. (Fig: 11)

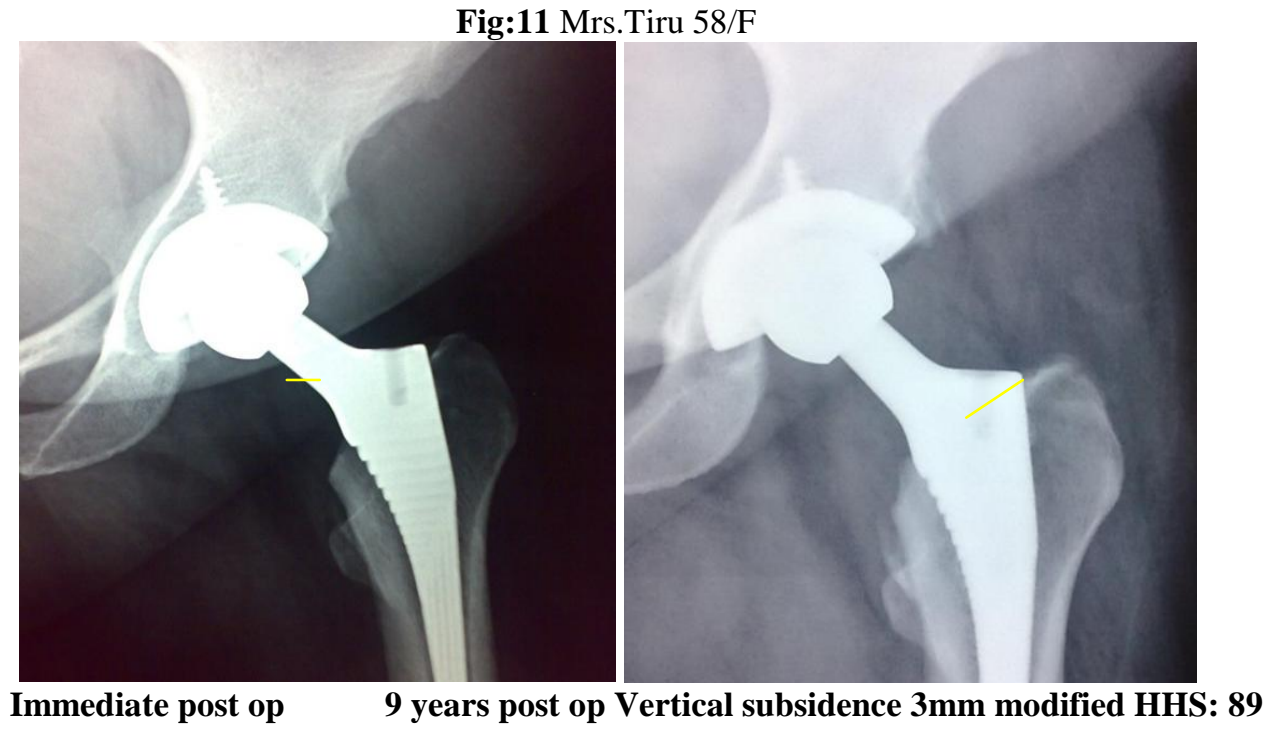


4.2.4: Endosteal Bone Apposition: The development of Endosteal bone formation in the form of spot-welds. The typical pattern of condensation against the medial distal area, in Gruen zone $6 \mathrm{~A}^{9}$. These spot-welds appeared in the $1^{\text {st }}$ year and present till the $8^{\text {th }}$ year follow up. In this study $3(7.5 \%)$ hips had Endosteal bony ingrowth at the latest follow up. (Fig: 12)

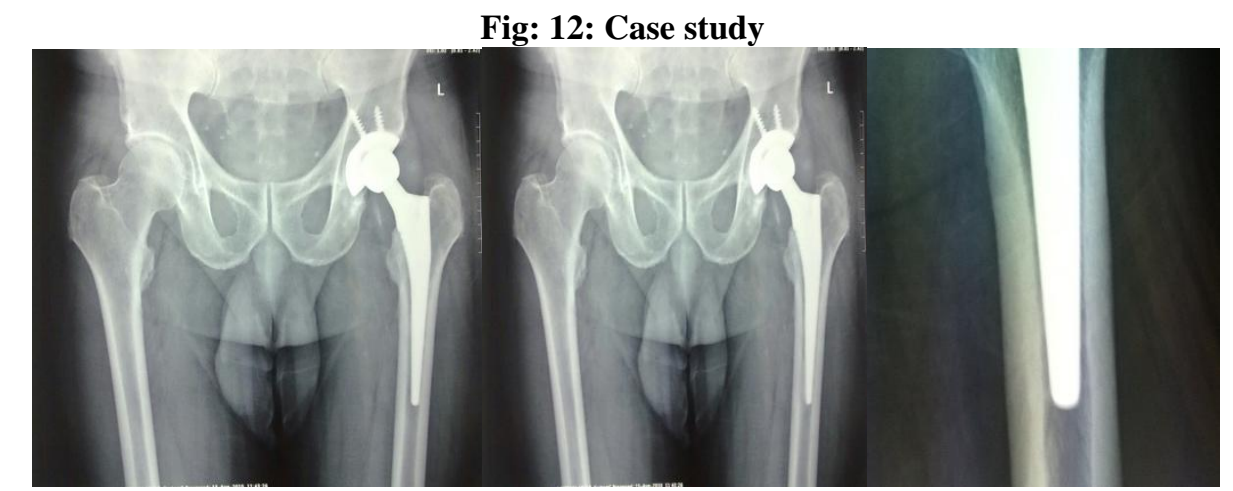

Immediate post op following Lt THR8 years post op showing Endosteal bone apposition distally

4.2.5: Heterotopic Ossification: Heterotopic bone when present was graded according to the classification of Brooker et al. ${ }^{6}$ In this series we had 0 hips which had no heterotopic ossification.

4.2.6: Bone Reactive Lines: There were very few reactive lines in bone around the HA-coated parts of the femoral stem (Gruen zones 6A). but such lines did not extend distally for more than $1.5 \mathrm{~cm}$. more often there was a small triangle of increased bone density in this area. Reactive lines were regularly seen around the distal non coated part of the stem. In this latest follow up $3(7.5 \%)$ had bone reactive lines.

4.2.7: Periosteal Bone Reaction: Periosteal or cortical bone remodeling reactions were seen in a 2(5\%) cases after HA-coated total hip replacement. We recognized less common asymmetrical type of pattern of remodeling. The asymmetrical type was due to localized periosteal reactions medially or laterally on the femur near the tip of the stem. Reactive remodeling appeared to be more common when there was a very tight fit of the stem in the femoral canal.

4.2.8: Pedestal Formation: No evidence of pedestal formation found during the follow up.

4.2.9: Calcar Resorption: Radiologically No evidence of calcar resorption found during the latest follow up.

4.2.10: Osteoporosis: Radiological osteoporosis was rare and was not seen in any patients during the latest follow up.

4.2.11: Osteolysis: No evidence of Osteolysis found during the latest follow up.

4.2.12: Polyethylene Wear: No evidence of polyethylene wear found during the follow up.

\section{Conclusion}

We have attempted to do a Midterm (7-9 years) follow up of un-cemented total hip arthroplasty. From this study we had arrived at the following conclusion.

- Un-cemented primary hydroxyapatite-coated total hip arthroplasty is a reliable procedure resulting in excellent implant fixation and a predictable clinical outcome in the medium term.

- The Modified Harris hip score is a very useful scoring system in assessing total hip replacement and showed high validity and reliability. We have $97.5 \%$ excellent/good results in our series.

- $80 \%$ of patients had radiologically silent hips with excellent clinical score.

- With Hydroxyapatite coated stem, there is no clinically significant anterior thigh pain due to good stem stability, in a midterm analysis.

- Intra-operative fissures (5\%) are not related to the stem design, but can be avoided with careful and less aggressive broaching of the femoral canal. It has not affected the clinical and radiological outcome.

\section{References}

[1]. Nas.Ser.Eftekhar. Principles of total hip arthroplasty 1978:p143-146.

[2]. August A.C.et al. (1986) The McKee-Farrar hip arthroplasty, J Bone Joint Surg ,68-B(4), 520-27.

[3]. Waugh, w.JohnCharnley: the man and the hip: p102-144

[4]. Richard S Snell: clinical anatomy for medical students. $9^{\text {th }}$ edition: p564-568

[5]. Campbell's operative Orthopaedics. International $12^{\text {th }}$ edition.p234-459.

[6]. Brooker AF, Bowermann JW, Railey LH, Ectopic ossification following total hip replacement, incidence and classification,J Bone Joint Surg Am. 1973 Dec;55(8):1629-1632.

[7]. Woo RY and Moorey BJ. Dislocation following total hip replacement, J DtschArzteblInt 2014; 111: 884-90.

[8]. M.A.Ali khan, P.H.Brankenbury, dislocation following total hip replacement, J Bone Joint Surg Br 77:11-17. 
[9]. Harris WH, traumatic arthritis of the hip after dislocation and acetabular fractures: treatment by mold arthroplasty. An end result study using a new method of result evaluation. J Bone Joint Surg Am:1969;51:737-55

[10]. Gruen TA, Mc Neice GM. Modes of failure of uncemented type of femoral components. A radiographic analysis of loosening, ClinOrthopRelat Res 1979;141:17-27.

[11]. Engh's criteria for loosening of uncemented femoral component. A retrospective study of total hip arthroplasty,ClinOrthop1990; vol-4:issue 1;62-66.

[12]. Mont MA, Maar D.C, Krackow KA, total hip replacement without cement for non- inflammatory arthritis in patients who are less than 40 years old, J Bone Joint Surg Br 1993 75(5):740-751.

[13]. Ilchmann T, Eingartner c, Hegar K, femoral subsidence assessment after hip replacement; an experimental study, $\quad$ J Bone Joint Surg Br. 2006;111(3):361-9.

[14]. Zicat B, Engh CA, Gokcen E, pattern of osteolysis around total hip components inserted with and without cement. J Bone Joint Surg Am 1995;77(3):432-9.

[15]. Ragsdale et al, radiological evaluation of femoral components following primary total hip arthroplasty. J Arthroplasty 1994; 9(4):286-94.

[16]. Meding JB, Ritter MA, Keating EM, Faris PM, clinical and radiological evaluation of long stem femoral components following revision total hip arthroplasty. J Arthroplasty 2014;9(4):399-408.

[17]. Aldinger PR, Breusch SJ, Lukoschek M, a 10-15 year follow up of the uncementedsportorno stem. J Bone Joint Surg Br 2003;85(2):209-14.

[18]. Xian-fengLou et al, J Zhejiang,JUniv Sci,2007 jan 8(1):76-80.

[19]. R.Dattani, femoral osteolysis following total hip replacement, J Bone Joint Surg Br 2007;83(97a):312-316.

[20]. Young-Hooet al, uncemented porous coated anatomic total hip replacement results at six years in a consecutive series, J Bone Joint Surg Br 1993;75-B:6-14

[21]. Jean-Pierre Vidalainet al twenty year results of the cementless corail stem, Inter Orthop(sicot)(2011)35:189-194

[22]. Jasty M, Webster W and Harris W, limb length inequality in a series of 85 total hip replacements, J Clinic Orthop,dec; 1996:167171.

[23]. Martin Clausset al Prospective five year subsidence analysis of a cementless fully hydroxyapatite-coated femoral hip arthroplasty component J Hip Int 2014;24(1):91-97.

[24]. Rudolph G.T.Geesinket al six year results of hydroxyapatite coated total hip replacement J Bone Joint Surg Br 1995;77-B: 534-47.

[25]. JC Thesis, C Ball, medium-term results of cement less hydroxyapatite coated primary total hip arthroplasty: a clinical and radiological outcome, J OrthopSurg 2003:11(2):159-165. 